\title{
SPECTRAL REPRESENTATION OF CERTAIN ONE-PARAMETRIC FAMILIES OF SYMMETRIC OPERATORS IN HILBERT SPACE
}

\author{
BY \\ A. E. NUSSBAUM( $\left.{ }^{1}\right)$
}

\begin{abstract}
It is proved that if a one-parameter family of symmetric operators acting in a Hilbert space has the semigroup property on a dense linear manifold and is weakly continuous, then the operators are essentially selfadjoint and permute in the sense of permuting spectral projections of the selfadjoint extensions. It follows from this that the operators have a joint spectral integral representation.
\end{abstract}

1. Introduction. If $\left\{T_{\alpha}\right\}, \alpha>0$, is a semigroup of selfadjoint operators acting in a Hilbert space $\mathfrak{F}$, i.e. $T_{\alpha} T_{\beta}=T_{\alpha+\beta}$ for all $\alpha$ and $\beta>0$, and if for every $x \in \bigcap_{\alpha>0} D\left(T_{\alpha}\right)$ (if $S$ is an operator acting in $\mathfrak{H}$ we denote by $D(S)$ its domain) $\left(T_{\alpha} x, x\right)$ is either a bounded or measurable function of $\alpha$ in some interval, then there exists a unique positive selfadjoint operator $T$ in $\mathfrak{F}$ such that $T_{\alpha}=T^{\alpha}$ for all $\alpha>0$. This theorem is well known in case the operators are bounded. A particularly simple and elegant proof was given by B. v. Sz. Nagy (cf. [3, p. 73]). Using the basic idea of Nagy's proof, A. Devinatz [2] extended the theorem to unbounded operators. Except for minor changes the proof is as follows: The conditions on $\left(T_{\alpha} x, x\right)$ imply [3, p. 73] that the function $\alpha \rightarrow\left(T_{\alpha} x, y\right)$ is continuous for every $x$ and $y$ in $\bigcap_{\alpha>0} D\left(T_{\alpha}\right)$. Let $T_{1}=\int_{0}^{\infty} \lambda d E(\lambda)$ be the canonical spectral representation of $T_{1}$. Since $T_{1}=T_{1 / 2}^{2}$ and a positive selfadjoint operator has a unique positive selfadjoint square root it follows that

$$
T_{1 / 2}=\int_{0}^{\infty} \lambda^{1 / 2} d E(\lambda)
$$

Repeating this argument we get that

$$
T_{m / 2^{n}}=\int_{0}^{\infty} \lambda^{m / 2^{n}} d E(\lambda)=T_{1}^{m / 2^{n}},
$$

that is, $T_{\alpha}=T_{1}^{\alpha}$ for all $\alpha$ of the form $\alpha=m / 2^{n}$, where $m$ and $n$ are positive integers. From this follows, using the fact that $\alpha \rightarrow\left(T_{\alpha} x, y\right)$ and $\alpha \rightarrow\left(T_{1}^{\alpha} x, y\right)$ are continuous for every $x$ and $y$ in $D=\bigcap_{\alpha>0} D\left(T_{\alpha}\right)$, that $T_{\alpha} x=T_{1}^{\alpha} x$ for all $x \in D$ and $\alpha>0$. We have tacitly assumed that $D=\bigcap_{\alpha>0} D\left(T_{1}^{\alpha}\right)$. This follows from the fact that if

Received by the editors December 15, 1969.

AMS 1968 subject classifications. Primary 4750, 4730.

Key words and phrases. Symmetric operators, Hilbert space, semigroup, unbounded operators, spectral representation.

(1) This work was in part supported by National Science Foundation Grant NSF GP-9657.

Copyright (C) 1970, American Mathematical Society 
$\alpha<m / 2^{n}$ then $D\left(T_{m / 2^{n}}\right) \subset D\left(T_{\alpha}\right)$ and $D\left(T_{1}^{m / 2^{n}}\right) \subset D\left(T_{1}^{\alpha}\right)$ since $T_{m / 2^{n}}=T_{m / 2^{n}-\alpha} T_{\alpha}$ and similarly $T_{1}^{m / 2^{n}}=T_{1}^{m / 2^{n}-\alpha} T_{1}^{\alpha}$. Hence

$$
D=\bigcap_{\alpha>0} D\left(T_{\alpha}\right)=\bigcap D\left(T_{m / 2^{n}}\right)=\bigcap D\left(T_{1}^{m / 2^{n}}\right)=\bigcap_{\alpha>0} D\left(T_{1}^{\alpha}\right),
$$

where in the intersection of the two middle terms $m$ and $n$ ranges over the set of positive integers. Let $E_{n}=E([0, n])$, then $E_{n} \mathfrak{S} \subset D$. If now $\alpha>0$ and $x \in D\left(T_{1}^{\alpha}\right)$, then $E_{n} x \rightarrow x$ and $T_{\alpha} E_{n} x=T_{1}^{\alpha} E_{n} x=E_{n} T_{1}^{\alpha} x \rightarrow T_{1}^{\alpha} x$ as $n \rightarrow \infty$. Therefore, since $T_{\alpha}$ is a closed operator, $x \in D\left(T_{\alpha}\right)$ and $T_{\alpha} x=T_{1}^{\alpha} x$, i.e. $T_{1}^{\alpha} \subset T_{\alpha}$. This implies that $T_{\alpha}=T_{1}^{\alpha}$.

The purpose of this paper is to significantly extend this theorem as follows:

Let $\left\{S_{\alpha}\right\}, 0<\alpha<a$, be a one-parametric family of symmetric operators in $\mathfrak{S}$, where $0<a \leqq \infty$, and $D$ be a dense linear manifold in $\mathfrak{H}$ contained in $D\left(S_{\alpha} S_{\beta}\right)$ for all $\alpha, \beta$ such that $0<\alpha+\beta<$ a. Suppose that

$$
S_{\alpha} S_{\beta} x=S_{\alpha+\beta} x
$$

for all $x \in D$ and $\alpha, \beta$ such that $0<\alpha+\beta<a$ and $\left(S_{\alpha} x, x\right)$ is either a bounded or measurable function of $\alpha$ in some interval for every $x \in D$. Then the operators $S_{\alpha}$ are essentially selfadjoint and there exists a unique positive selfadjoint operator $T$ such that $\bar{S}_{\alpha}=T^{\alpha}$ for all $\alpha .\left(\bar{S}_{\alpha}\right.$ denotes the closure of $S_{\alpha}$.)

Note that the theorem is not trivial even if we assume that $a=\infty$, the operators $S_{\alpha}$ are all selfadjoint and $D$ is invariant under every $S_{\alpha}$. At least the proof of NagyDevinatz fails for several reasons. For example we cannot conclude that $T_{1}=T_{1 / 2}^{2}$ from the sole fact that $T_{1} x=T_{1 / 2}^{2} x$ for all $x$ in $D$.

We shall indicate some applications of our theorem, others and extensions of our result will be discussed in another publication.

\section{Preliminary results.}

Proposition 1. If $A$ and $B$ are permuting selfadjoint operators in a Hilbert space $\mathfrak{S}$ (i.e., $A$ and $B$ have permuting spectral projections) and $A x=B x$ for all $x$ in a dense set $D$, then $A=B$.

Proof. The proposition is certainly well known, but for the sake of completeness we shall give a proof. Let $\{E(\sigma)\}$ and $\{F(\tau)\}$ be the canonical spectral measures of $A$ and $B$, respectively. For every positive integer $n$ let $E_{n}=E([-n, n])$ and $F_{n}=$ $F([-n, n])$. Let $P_{n}=E_{n} F_{n}$. Then $P_{n} \rightarrow I$ (strongly) as $n \rightarrow \infty$ since $E_{n} \rightarrow I$ and $F_{n} \rightarrow I$ as $n \rightarrow \infty$. ( $I$ denotes the identity operator in $\left.\mathfrak{S}_{\text {.) }}\right)$ Let $x \in D(B)$, then $P_{n} x \in D(B)$ and $P_{n} x \in D(A)$ (this is true for any $x \in \mathfrak{S}$ for $F_{n} x \in D(B)$ and $E_{n} x \in D(A)$ for all $x \in \mathfrak{S}$ and $E_{n} B F_{n} \subset B E_{n} F_{n}=B P_{n}$ and $\left.F_{n} A E_{n} \subset A F_{n} E_{n}=A E_{n} F_{n}=A P_{n}\right)$. Hence for every $y \in D$

$$
\left(B P_{n} x, y\right)=\left(P_{n} x, B y\right)=\left(P_{n} x, A y\right)=\left(A P_{n} x, y\right) .
$$

Since $D$ is dense in $\mathfrak{S}$ we conclude that $A P_{n} x=B P_{n} x$ for all $n$. Now $P_{n} x \rightarrow x$ and $A P_{n} x=B P_{n} x=P_{n} B x \rightarrow B x$ as $n \rightarrow \infty$. Therefore $x \in D(A)$ and $A x=B x$, since $A$ is closed. Thus, $B \subset A$. Similarly $A \subset B$ and therefore $A=B$. 
The next lemma appears to be new and is important in the proof of the main theorem. We recall that a regular point of an operator $A$ in $\mathfrak{S}$ is a complex number $a$ such that $(A-a I)^{-1}$ exists and is bounded (but the domain of $(A-a I)^{-1}$ is not assumed to be $\mathfrak{S}$ or even dense in $\mathfrak{S}$ ). We denote by $\Lambda(A)$ the set of regular points of $A$ and by $R(A)$ and $N(A)$ the range and the nullspace of $A$, respectively.

LemMa 1. Let $S_{1}$ and $S_{2}$ be two symmetric operators in a Hilbert space $\mathfrak{S}_{2}$ and suppose that each operator has equal deficiency indices. If

(1) $S_{1} S_{2} x=S_{2} S_{1} x$ for all $x \in D$, where $D$ is a linear manifold contained in $D\left(S_{1} S_{2}\right) \cap D\left(S_{2} S_{1}\right)$ and

(2) $\left(S_{1}-a_{1} I\right)\left(S_{2}-a_{2} I\right) D$ is dense in $\mathfrak{S}$ for some $a_{1} \in \Lambda\left(S_{1}\right)$ and $a_{2} \in \Lambda\left(S_{2}\right)$, then $T_{1}=\bar{S}_{1}$ and $T_{2}=\bar{S}_{2}$ are selfadjoint and permute.

Proof. We first note that $a_{i} \in \Lambda\left(T_{i}\right)$ and $R\left(T_{i}-a_{i} I\right)$ is closed in $\mathfrak{S}$ for $i=1,2$. The first statement is obvious and the second follows from the fact that $R\left(T_{i}-a_{i} I\right)$ is the domain of the closed, continuous operator $\left(T_{i}-a_{i} I\right)^{-1}$. Next, $R\left(T_{i}-a_{i} I\right)=\mathfrak{S}_{\mathrm{E}}$ for $i=1$, 2. For suppose that $y \neq 0, y \in R\left(T_{1}-a_{1} I\right)^{\perp} \quad\left(\right.$ or $\left.y \in R\left(T_{2}-a_{2} I\right)^{\perp}\right)$. ( $M^{\perp}$ denotes the orthogonal complement of a linear manifold $M$ in $\mathfrak{S}$.) Then $\left(\left(S_{1}-a_{1} I\right)\left(S_{2}-a_{2} I\right) x, y\right)=0$ for all $x \in D$. This contradicts the hypothesis (2).

$R\left(T_{i}-a_{i} I\right)=\mathfrak{H}$ implies $N\left(T_{i}^{*}-\bar{a}_{i} I\right)=R\left(T_{i}-a_{i} I\right)^{\perp}=\{0\}$ and this shows that the deficiency indices of $T_{i}$ are $(0,0)$, i.e. $T_{1}$ and $T_{2}$ are selfadjoint.

We now show that $T_{1}$ and $T_{2}$ permute. Let $x$ be an element of $D$ and

$$
y=\left(T_{1}-a_{1} I\right)\left(T_{2}-a_{2} I\right) x .
$$

Then

$$
y=\left(T_{2}-a_{2} I\right)\left(T_{1}-a_{1} I\right) x \text { by }(1),
$$

and therefore

$$
\left(T_{1}-a_{1} I\right)^{-1}\left(T_{2}-a_{2} I\right)^{-1} y=\left(T_{2}-a_{2} I\right)^{-1}\left(T_{1}-a_{1} I\right)^{-1} y=x .
$$

From this it follows, since the set of $y$ 's is dense in $\mathfrak{S}$ by (2) and the operators $\left(T_{1}-a_{1} I\right)^{-1}$ and $\left(T_{2}-a_{2} I\right)^{-1}$ are bounded operators with domain $\mathfrak{S}$, that

$$
\left(T_{1}-a_{1} I\right)^{-1}\left(T_{2}-a_{2} I\right)^{-1}=\left(T_{2}-a_{2} I\right)^{-1}\left(T_{1}-a_{1} I\right)^{-1} .
$$

This implies that $\left(T_{1}-a_{1} I\right)^{-1}$ permutes with $T_{2}$. This in turn implies by the spectral theorem that the spectral projections of $T_{2}$ permute with $\left(T_{1}-a_{1} I\right)^{-1}$ and hence with $T_{1}$. Therefore, again by the spectral theorem, it follows that the spectral projections of $T_{2}$ permute with the spectral projections of $T_{1}$.

\section{Main result.}

THEOREM 1. Let $\left\{S_{\alpha}\right\}, 0<\alpha<a,(0<a \leqq \infty)$ be a one-parametric family of symmetric operators in a Hilbert space $\mathfrak{S}$ and $D$ be a dense linear manifold in $\mathfrak{S}$ such that

(1) $D \subset D\left(S_{\alpha} S_{\beta}\right)$ for all $\alpha, \beta$ such that $0<\alpha+\beta<a$,

(2) $S_{\alpha} S_{\beta} x=S_{\alpha+\beta} x$ for all $x \in D$ and $\alpha, \beta$ such that $0<\alpha+\beta<a$, and 
(3) for every $x \in D,\left(S_{\alpha} x, x\right)$ is a bounded or Lebesgue measurable function of $\alpha$ in some interval.

Then the operators $S_{\alpha}, 0<\alpha<a$, are essentially selfadjoint and there exists a unique positive selfadjoint operator $T=\int_{0}^{\infty} \lambda d E(\lambda)$ such that

\section{If furthermore}

$$
\bar{S}_{\alpha}=T^{\alpha}=\int_{0}^{\infty} \lambda^{\alpha} d E(\lambda) \text { for all } 0<\alpha<a .
$$

(4) $\left(S_{\alpha} x, x\right) \rightarrow\|x\|^{2}$ as $\alpha \rightarrow 0+$ for every $x$ in a dense linear manifold $D_{0} \subset D$, then $T>0$ and $\bar{S}_{\alpha}=e^{\alpha A}$ for $0<\alpha<a$, where

$$
A=\log T=\int_{0}^{\infty} \log \lambda d E(\lambda) .
$$

Proof. The function $f_{x}(\alpha)=\log \left(S_{\alpha} x, x\right), x \in D$, is a midpoint convex function on the interval $0<\alpha<a$ for

$$
\begin{aligned}
f_{x}((\alpha+\beta) / 2) & =\log \left(S_{(\alpha+\beta) / 2} x, x\right)=\log \left(S_{\alpha / 2} x, S_{\beta / 2} x\right) \leqq \log \left\|S_{\alpha / 2} x\right\|+\log \left\|S_{\beta / 2} x\right\| \\
& \leqq \frac{1}{2} \log \left(S_{\alpha} x, x\right)+\frac{1}{2} \log \left(S_{\beta} x, x\right)=\frac{1}{2} f_{x}(\alpha)+\frac{1}{2} f_{x}(\beta) .
\end{aligned}
$$

This, together with condition (3), implies that the function $f_{x}(\alpha)$ and hence $\left(S_{\alpha} x, x\right)$ is continuous on the interval $0<\alpha<a$, as was noted by Nagy [3, p. 73]. From this follows that the function $\alpha \rightarrow\left(S_{\alpha} x, y\right)$ is continuous on the interval $0<\alpha<a / 2$ for every $x \in D$ and $y \in \mathfrak{S}$ because

as $\alpha \rightarrow \beta$.

$$
\begin{aligned}
\left|\left(S_{\alpha} x, y\right)-\left(S_{\beta} x, y\right)\right|^{2} & \leqq\left\|S_{\alpha} x-S_{\beta} x\right\|^{2}\|y\|^{2} \\
& \leqq\left[\left(S_{2 \alpha} x, x\right)-2\left(S_{\alpha+\beta} x, x\right)+\left(S_{2 \beta} x, x\right)\right]\|y\|^{2} \rightarrow 0
\end{aligned}
$$

For every $0<\alpha<a$ let $A_{\alpha}$ be the restriction of $S_{\alpha}$ to $D \cup \bigcup_{0<t<a-\alpha} S_{t} D$. Then clearly $A_{\alpha}, 0<\alpha<a$, are symmetric operators in $\mathfrak{S}_{2}$ which satisfy conditions (1)-(3) with $S_{\alpha}$ replaced by $A_{\alpha}$. Moreover $A_{\alpha}$ is positive for every $0<\alpha<a$. Indeed, if $0<\alpha<a$, and $x \in D\left(A_{\alpha}\right)$ then either $x \in D$ or $x=S_{t} y$, where $y \in D$ and $0<t<a-\alpha$. In the first case

and in the latter

$$
\left(A_{\alpha} x, x\right)=\left(S_{\alpha} x, x\right)=\left\|S_{\alpha / 2} x\right\|^{2} \geqq 0
$$

$$
\left(A_{\alpha} x, x\right)=\left(S_{\alpha+t} y, S_{t} y\right)=\left\|S_{\alpha / 2+t} y\right\|^{2} \geqq 0 .
$$

Since $A_{\alpha}$ is positive it has a positive selfadjoint extension, for example the Friedrich extension. For every $0<\alpha<a$ let $T_{\alpha}$ be any positive selfadjoint extension of $A_{\alpha}$. Clearly we may write $T_{\alpha}$ (uniquely) of the form

$$
T_{\alpha}=\int_{0}^{\infty} t^{\alpha} d E_{\alpha}(t)
$$

where $\left\{E_{\alpha}(\sigma)\right\}$ is a spectral measure on $[0, \infty)\left({ }^{2}\right)$.

$\left.{ }^{2}\right)$ If $\{E(\sigma)\}$ is the canonical spectral measure of $T_{\alpha}$ on $[0, \infty)$, then $E_{\alpha}(\sigma)=E\left(\sigma^{1 / \alpha}\right)$, where $\sigma^{1 / \alpha}=\left\{t \geqq 0 \mid t^{\alpha} \in \sigma\right\}$. 
Next we show that for every $x \in D$ and $y \in \mathfrak{F}$ there exists a bounded Radon measure $\mu_{x, y}$ on $[0, \infty)$ such that

$$
\left(S_{\alpha} x, y\right)=\int_{0}^{\infty} t^{\alpha} d \mu_{x, y}(t) \text { for all } 0<\alpha<a / 2
$$

Choose $0<\alpha_{0}<a$ arbitrarily. For $x \in D$ and $y \in \mathfrak{S}$ let $\mu_{x, y}^{(n)}$ be the Radon measure determined by $\mu_{x, y}^{(n)}(\sigma)=\left(E_{\alpha_{0} / 2^{n}}(\sigma) x, y\right), n=1,2, \ldots$ Since $\left\|\mu_{x, y}^{(n)}\right\| \leqq\|x\|\|y\|$ for all $n$ there exists a subsequence $\left\{\mu_{x, y}^{\left(n_{r}\right)}\right\}$ of $\left\{\mu_{x, y}^{(n)}\right\}$ which converges to a bounded Radon measure $\mu_{x, y}$ on $[0, \infty)$ in the $w^{*}$-topology of the conjugate space of $C_{0}([0, \infty)$ ) (the space of complex-valued continuous functions on $[0, \infty)$ vanishing at infinity).

If $0<\alpha<a$ is of the form $\alpha=\left(k / 2^{n}\right) \alpha_{0}$, where $k$ and $n$ are positive integers, and $x \in D$ and $y \in \mathfrak{H}$, then

$$
\left(S_{\alpha} x, y\right)=\left(A_{\alpha} x, y\right)=\left(A_{\alpha_{0} / 2^{n}}^{k} x, y\right)=\left(T_{\alpha_{0} / 2^{n}}^{k} x, y\right)=\int_{0}^{\infty} t^{\alpha} d \mu_{x, y}^{(n)}(t)
$$

and

$$
\left\|S_{\alpha} x\right\|^{2}=\left\|A_{\alpha} x\right\|^{2}=\left\|A_{\alpha_{0} / 2^{n}}^{k} x\right\|^{2}=\left\|T_{\alpha_{0} / 2^{n}}^{k} x\right\|^{2}=\int_{0}^{\infty} t^{2 \alpha} d \mu_{x, x}^{(n)}(t) .
$$

Thus, if $0<\alpha<a$ is of the form $\alpha=\left(k / 2^{m}\right) \alpha_{0}$, where $k$ and $m$ are positive integers and $x \in D, y \in \mathfrak{H}$, then

$$
\left(S_{\alpha} x, y\right)=\int_{0}^{\infty} t^{\alpha} d \mu_{x, y}^{(n)}(t) \text { and }\left\|S_{\alpha} x\right\|^{2}=\int_{0}^{\infty} t^{2 \alpha} d \mu_{x, x}^{(n)}(t)
$$

for all sufficiently large $n$. If now $B$ is any positive number and $\alpha<\alpha^{\prime}<a$ is another number of the form $\alpha^{\prime}=\left(k^{\prime} / 2^{m^{\prime}}\right) \alpha_{0}$, where $k^{\prime}$ and $m^{\prime}$ are positive integers, then

$$
\begin{aligned}
\left|\left(S_{\alpha} x, y\right)-\int_{0}^{B} t^{\alpha} d \mu_{x, y}^{(n)}(t)\right| & \leqq \int_{B}^{\infty} t^{\alpha-\alpha^{\prime}} t^{\alpha^{\prime}} d\left|\mu_{x, y}^{(n)}\right|(t) \\
& \leqq B^{\alpha-\alpha^{\prime}} \int_{0}^{\infty} t^{\alpha^{\prime}} d\left|\mu_{x, y}^{(n)}\right|(t) \\
& \leqq B^{\alpha-\alpha^{\prime}}\left(\int_{0}^{\infty} t^{2 \alpha^{\prime}} d \mu_{x, x}^{(n)}(t)\right)^{1 / 2}\left(\int_{0}^{\infty} d \mu_{y, y}^{(n)}(t)\right)^{1 / 2} \\
& \leqq B^{\alpha-\alpha^{\prime}}\left\|S_{\alpha^{\prime}} x\right\|\|y\|
\end{aligned}
$$

for all sufficiently large $n$. Hence if we choose $B$ to be a point at which $\mu_{x, y}$ is continuous (i.e. $\mu_{x, y}(\{B\})=0$ ), let $n=n_{r}$ and let $r \rightarrow \infty$, we obtain the inequality

$$
\left|\left(S_{\alpha} x, y\right)-\int_{0}^{B} t^{\alpha} d \mu_{x, y}(t)\right| \leqq B^{\alpha-\alpha^{\prime}}\left\|S_{\alpha^{\prime}} x\right\|\|y\| .
$$

From this follows, if we let $B \rightarrow \infty$ through a sequence of points at which $\mu_{x, y}$ is continuous, that

$$
\left(S_{\alpha} x, y\right)=\int_{0}^{\infty} t^{\alpha} d \mu_{x, y}(t)
$$


Since both sides of this equation are continuous functions of $\alpha$ in the interval $0<\alpha<a / 2$ we conclude that

$$
\left(S_{\alpha} x, y\right)=\int_{0}^{\infty} t^{\alpha} d \mu_{x, y}(t)
$$

for all $0<\alpha<a / 2, x \in D$ and $y \in \mathfrak{S}$. Let $\nu_{x, y}$ be the bounded Radon measure on the real line defined by $d \nu_{x, y}(s)=d \mu_{x, y}\left(e^{s}\right)$, then

$$
\left(S_{\alpha} x, y\right)=\int_{-\infty}^{\infty} e^{\alpha s} d v_{x, y}(s)
$$

for all $0<\alpha<a / 2, x \in D$ and $y \in \mathfrak{S}$.

We now prove that if $0<\alpha+\beta<a / 2$, then $\bar{S}_{\alpha}$ and $\bar{S}_{\beta}$ are selfadjoint and permute. Since $A_{\alpha} \subset S_{\alpha}$ and $A_{\beta} \subset S_{\beta}$ it is sufficient to show that $\bar{A}_{\alpha}$ and $\bar{A}_{\beta}$ are selfadjoint and permute. This we prove by applying Lemma 1 . Since $A_{\alpha}$ and $A_{\beta}$ are positive they both have equal deficiency indices and to prove our assertion it is sufficient by Lemma 1 to show that the set of vectors

$$
\left\{\left(A_{\alpha}-i I\right)\left(A_{\beta}-i I\right) x\right\},
$$

where $x$ varies over $D\left(A_{\alpha+\beta}\right)$, is dense in $\mathfrak{S}$. (Note that $D\left(A_{\alpha+\beta}\right)$ is contained in $D\left(A_{\alpha}\right), D\left(A_{\beta}\right)$ and $D\left(A_{\alpha} A_{\beta}\right)$.) Suppose $y \in \mathfrak{F}$ and

$$
\left(\left(A_{\alpha}-i I\right)\left(A_{\beta}-i I\right) x, y\right)=0 \text { for all } x \in D\left(A_{\alpha+\beta}\right) \text {. }
$$

Then in particular $\left(\left(A_{\alpha}-i I\right)\left(A_{\beta}-i I\right) S_{t} z, y\right)=0$ for all $z \in D$ and $0<t<a / 2-(\alpha+\beta)$. Then

$$
\left(\left(A_{\alpha+\beta+t}-i A_{\beta+t}-i A_{\alpha+t}-A_{t}\right) z, y\right)=\int_{-\infty}^{\infty} e^{t s}\left(e^{\alpha s}-i\right)\left(e^{\beta s}-i\right) d \nu_{z, y}(s)=0
$$

for all $0<t<a / 2-(\alpha+\beta)$ and every $z \in D$. From this follows by the uniqueness theorem for Laplace-Stieltjes integrals [5, p. 243] that the measure

$$
\left(e^{\alpha s}-i\right)\left(e^{\beta s}-i\right) d \nu_{z, y}(s)
$$

is zero for every $z \in D$. Hence $\nu_{z, y}=0$ for every $z \in D$. Therefore

for all $0<\gamma<a / 2$ and $x \in D$. But

$$
\left(A_{\gamma} x, y\right)=\int_{-\infty}^{\infty} e^{\gamma s} d v_{x, y}(s)=0
$$

$$
\left(\left(A_{\alpha}-i I\right)\left(A_{\beta}-i I\right) x, y\right)=\left(A_{\alpha+\beta} x, y\right)-i\left(A_{\beta} x, y\right)-i\left(A_{\alpha} x, y\right)-(x, y)=0
$$

for all $x \in D$. Hence $(x, y)=0$ for all $x \in D$. Therefore $y=0$, since $D$ is dense in $\mathfrak{B}$. This shows that $\bar{A}_{\alpha}=\bar{S}_{\alpha}$ and $\bar{A}_{\beta}=\bar{S}_{\beta}$ are selfadjoint and permute if $0<\alpha+\beta<a / 2$. Since $A_{\alpha} \subset T_{\alpha}$ and $T_{\alpha}$ is selfadjoint it also follows that

$$
\bar{S}_{\alpha}=\bar{A}_{\alpha}=T_{\alpha} \text { for } 0<\alpha<a / 2 .
$$

Now let $0<b<a / 2$ be arbitrary but fixed and $\{E(\sigma)\}$ be the spectral measure $\left\{E_{b}(\sigma)\right\}$ on $[0, \infty)$. 
Let $T=\int_{0}^{\infty} \lambda d E(\lambda)$, then $\bar{S}_{b}=T_{b}=\int_{0}^{\infty} \lambda^{b} d E(\lambda)=T^{b}$. Now $\bar{S}_{b / 2}$ is a positive selfadjoint operator which permutes with $\bar{S}_{b}$. Hence $\bar{S}_{b / 2}^{2}$ permutes with $\bar{S}_{b}$. But $\bar{S}_{b / 2}^{2} x=S_{b / 2}^{2} x=S_{b} x=\bar{S}_{b} x$ for all $x \in D$. Hence $\bar{S}_{b / 2}^{2}=\bar{S}_{b}$ by Proposition 1 . From this follows, since a positive selfadjoint operator has a unique positive selfadjoint square root, that $\bar{S}_{b / 2}=\bar{S}_{b}^{1 / 2}$. That is,

$$
\bar{S}_{b / 2}=\int_{0}^{\infty} \lambda^{b / 2} d E(\lambda)=T^{b / 2} .
$$

Continuing this way we get that

$$
\bar{S}_{b / 2^{n}}=\int_{0}^{\infty} \lambda^{b / 2^{n}} d E(\lambda)=T^{b / 2^{n}}
$$

for all positive integers $n$. Suppose that $0<\alpha<a / 2$ is of the form $\alpha=\left(k / 2^{n}\right) b$, where $k$ and $n$ are positive integers. $\bar{S}_{\alpha}$ and $\bar{S}_{b / 2^{n}}$ permute and therefore $\bar{S}_{\alpha}$ and $\bar{S}_{b / 2^{n}}^{k}$ permute. Moreover

$$
\bar{S}_{\alpha} x=S_{\alpha} x=S_{b / 2^{n}}^{k} x=\bar{S}_{b / 2^{n}}^{k} x \text { for all } x \in D,
$$

hence $\bar{S}_{\alpha}=\bar{S}_{b / 2^{n}}^{k}$ by Proposition 1. But

$$
\bar{S}_{b / 2^{n}}^{k}=\int_{0}^{\infty} \lambda^{k b / 2^{n}} d E(\lambda)=\int_{0}^{\infty} \lambda^{\alpha} d E(\lambda)=T^{\alpha} .
$$

Thus $\bar{S}_{\alpha}=T^{\alpha}$ for all $0<\alpha<a / 2$ of the form $\alpha=\left(k / 2^{n}\right) b$, where $k$ and $n$ are positive integers.

Next we prove that $\bar{S}_{\alpha}=T^{\alpha}$ for all $0<\alpha<a / 2$. First we note that $D \subset D\left(T^{\alpha}\right)$ for all $0<\alpha<a$. Indeed, if $0<\alpha<a$, choose positive integers $k$ and $n$ such that $\alpha<\beta=\left(k / 2^{n}\right) b<a$. Then

$$
S_{\beta / 2}^{2} \subset \bar{S}_{\beta / 2}^{2}=\left(T^{\beta / 2}\right)^{2}=T^{\beta} .
$$

Hence $D \subset D\left(T^{\beta}\right) \subset D\left(T^{\alpha}\right)$. If now $x \in D$ and $y \in \mathfrak{F}$, then

$$
\left(T^{\alpha} x, y\right)=\int_{0}^{\infty} \lambda^{\alpha} d(E(\lambda) x, y) \text { and }\left(\bar{S}_{\alpha} x, y\right)=\left(S_{\alpha} x, y\right)
$$

are continuous functions on the interval $0<\alpha<a / 2$ which agree on a dense set. Hence $\left(\bar{S}_{\alpha} x, y\right)=\left(T^{\alpha} x, y\right)$ for all $x \in D, y \in \mathfrak{S}$ and $0<\alpha<a / 2$. Therefore, if $0<\alpha<a / 2$, $\bar{S}_{\alpha} x=T^{\alpha} x$ for all $x \in D$. Furthermore, $\bar{S}_{\alpha}$ permutes with the spectral measure $\{E(\sigma)\}$ and hence with $T^{\alpha}$. Hence, by Proposition $1, \bar{S}_{\alpha}=T^{\alpha}$ for $0<\alpha<a / 2$.

Suppose $a<\infty$ and $a / 2 \leqq \alpha<a$. To show that $\bar{S}_{\alpha}=T^{\alpha}$ it is sufficient to show that $\bar{A}_{\alpha}=T^{\alpha}$ since $A_{\alpha} \subset S_{\alpha}$. To prove this we first show that $A_{\alpha}$ is essentially selfadjoint. Suppose that $A_{\alpha}^{*} y=i y$, then

$$
\left(A_{\alpha+\beta} x, y\right)=\left(A_{\beta} x, A_{\alpha}^{*} y\right)=-i\left(A_{\beta} x, y\right)
$$

for all $x \in D$ and $0<\beta<a-\alpha$. But if $x \in D$,

$$
A_{\alpha+\beta} x=A_{(\alpha+\beta) / 2}^{2} x=\left(T^{(\alpha+\beta) / 2}\right)^{2} x=T^{\alpha+\beta} x .
$$


Hence $\left(T^{\alpha+\beta} x, y\right)=-i\left(T^{\beta} x, y\right)$, i.e.

$$
\int_{0}^{\infty} \lambda^{\beta} \lambda^{\alpha} d(E(\lambda) x, y)=-i \int_{0}^{\infty} \lambda^{\beta} d(E(\lambda) x, y)
$$

for all $0<\beta<a-\alpha$ and every $x \in D$. Thus

$$
\int_{0<\lambda<\infty} \lambda^{\beta}\left(\lambda^{\alpha}+i\right) d(E(\lambda) x, y)=0
$$

for all $0<\beta<a-\alpha$ and every $x \in D$. From this follows, by the uniqueness theorem for Laplace-Stieltjes integrals [5, p. 243], that the measure $\left(\lambda^{\alpha}+i\right) d(E(\lambda) x, y)$, and hence $d(E(\lambda) x, y)$ restricted to the interval $0<\lambda<\infty$, is zero. Hence $(x, y)=$ $(E(\{0\}) x, y)=(x, E(\{0\}) y)$ for all $x \in D$. This implies that $E(\{0\}) y=y$. Hence $y \in D\left(T^{\alpha}\right)$ and $T^{\alpha} y=0$. But $A_{\alpha} \subset A_{\alpha / 2}^{2} \subset\left(T^{\alpha / 2}\right)^{2}=T^{\alpha}$ and therefore $A_{\alpha}^{*} \supset T^{\alpha}$. Hence $i y=A_{\alpha}^{*} y$ $=T^{\alpha} y=0$. Thus $A_{\alpha} \subset T^{\alpha}$ and $A_{\alpha}$ is essentially selfadjoint, hence $\bar{A}_{\alpha}=T^{\alpha}$.

Finally, if $x \in D$, then

$$
\left(S_{\alpha} x, x\right)=\left(T^{\alpha} x, x\right)=\int_{0}^{\infty} \lambda^{\alpha} d\|E(\lambda) x\|^{2} \rightarrow\|x\|^{2}-\|E(\{0\}) x\|^{2}
$$

as $\alpha \rightarrow 0+$. Therefore, if condition (4) is satisfied, $E(\{0\}) x=0$ for all $x \in D_{0}$ and hence $E(\{0\})=0$. It follows that $T>0$ and $\bar{S}_{\alpha}=e^{\alpha A}$ for all $\alpha$, where

$$
A=\log T=\int_{0}^{\infty} \log \lambda d E(\lambda)
$$

COROLlaRY 1. Let $\left\{S_{\alpha}\right\}, a<\alpha<b,(-\infty \leqq a<0<b \leqq \infty)$ be a one-parametric family of symmetric operators in a Hilbert space $\mathfrak{S}$ and $D$ be a dense linear manifold in $\mathfrak{S}$ such that

(1) $D \subset D\left(S_{\alpha} S_{\beta}\right)$ for all $\alpha, \beta$ such that $a<\alpha+\beta<b$,

(2) $S_{\alpha} S_{\beta} x=S_{\alpha+\beta} x$ for all $x \in D$ and $\alpha, \beta$ such that $a<\alpha+\beta<b$,

(3) $S_{0} x=x$ for all $x \in D$, and

(4) for every $x \in D,\left(S_{\alpha} x, x\right)$ is a bounded or Lebesgue measurable function of $\alpha$ in some interval.

Then the operators $S_{\alpha}, a<\alpha<b$, are essentially selfadjoint and there exists $a$ unique selfadjoint operator $A=\int_{-\infty}^{\infty} \lambda d E(\lambda)$ such that

$$
\bar{S}_{\alpha}=e^{\alpha A}=\int_{-\infty}^{\infty} e^{\alpha \lambda} d E(\lambda) \text { for } a<\alpha<b .
$$

Proof. (3) implies that $\bar{S}_{0}=I$ and by the preceding theorem there exist (unique) selfadjoint operators $A=\int_{-\infty}^{\infty} \lambda d E(\lambda)$ and $B=\int_{-\infty}^{\infty} \lambda d F(\lambda)$ such that

$$
\bar{S}_{\alpha}=e^{\alpha A}=\int_{-\infty}^{\infty} e^{\alpha \lambda} d E(\lambda) \text { for } 0 \leqq \alpha<b
$$

and

$$
\bar{S}_{\beta}=e^{\beta B}=\int_{-\infty}^{\infty} e^{\beta \lambda} d F(\lambda) \text { for } a<\beta \leqq 0
$$


To show that $A=B$ we first prove that $A$ and $B$ permute. If $x \in D, y \in D$ and $a<\beta<0$,

$$
\left(\bar{S}_{\alpha} \bar{S}_{\beta} x, y\right)=\int_{-\infty}^{\infty} e^{\alpha \lambda} d\left(E(\lambda) \bar{S}_{\beta} x, y\right)
$$

and

$$
\left(\bar{S}_{\alpha} \bar{S}_{\beta} x, y\right)=\left(\bar{S}_{\alpha} x, \bar{S}_{\beta} y\right)=\int_{-\infty}^{\infty} e^{\alpha \lambda} d\left(E(\lambda) x, \bar{S}_{\beta} y\right)
$$

for all $0 \leqq \alpha<b$. Hence by the uniqueness theorem for Laplace-Stieltjes integrals (loc. cit. p. 243) the measures $d\left(E(\lambda) x, \bar{S}_{\beta} y\right)$ and $d\left(E(\lambda) \bar{S}_{\beta} x, y\right)$ are equal for $x \in D$, $y \in D$ and $a<\beta<0$. Hence, for $x \in D$ and $y \in D$,

$$
\begin{aligned}
\left(E(\sigma) x, \bar{S}_{\beta} y\right) & =\int_{-\infty}^{\infty} e^{\beta \lambda} d(E(\sigma) x, F(\lambda) y) \\
& =\left(E(\sigma) \bar{S}_{\beta} x, y\right)=\left(\bar{S}_{\beta} x, E(\sigma) y\right) \\
& =\int_{-\infty}^{\infty} e^{\beta \lambda} d(F(\lambda) x, E(\sigma) y)
\end{aligned}
$$

for all Borel sets $\sigma$ and $a<\beta<0$. It follows again from the uniqueness theorem for Laplace-Stieltjes integrals that

$$
(E(\sigma) x, F(\tau) y)=(F(\tau) x, E(\sigma) y)
$$

for all Borel sets $\tau$ (and all Borel sets $\sigma$ and all $x, y$ in $D$ ). This implies, since $D$ is dense in $\mathfrak{S}$, that the spectral projections of $A$ and $B$ permute.

Let $0<\alpha<b$ and suppose that $a<-\alpha$. We shall prove that $\bar{S}_{-\alpha}=\bar{S}_{\alpha}^{-1}$, i.e. $e^{-\alpha B}=e^{-\alpha A} \cdot \bar{S}_{-\alpha}$ and $\bar{S}_{\alpha}^{-1}$ permute since $A$ and $B$ permute and if $y=S_{\alpha+\beta} x$, where $x \in D$ and $0<\beta<b-\alpha$,

$$
\bar{S}_{-\alpha} y=S_{-\alpha} S_{\alpha+\beta} x=S_{\beta} x=\bar{S}_{\alpha}^{-1} S_{\alpha} S_{\beta} x=\bar{S}_{\alpha}^{-1} y .
$$

But the set of all such $y$ is dense in $\mathfrak{S}$ for, if

$$
\int_{-\infty}^{\infty} e^{\beta \lambda} e^{\alpha \lambda} d(E(\lambda) x, z)=\left(S_{\alpha+\beta} x, z\right)=0
$$

for all $0<\beta<b-\alpha$ and $x \in D$, then once more from the uniqueness theorem for Laplace-Stieltjes integrals we obtain that the measure $e^{\alpha \lambda} d(E(\lambda) x, z)$ and hence $d(E(\lambda) x, z)$ is zero for all $x \in D$. It follows that $(x, z)=0$ for all $x \in D$ and hence $z=0$. Thus $\bar{S}_{-\alpha}$ and $\bar{S}_{\alpha}^{-1}$ permute and agree on a dense set. Hence by Proposition 1 , $\bar{S}_{-\alpha}=\bar{S}_{\alpha}^{-1}$.

Finally, if $a<-\alpha<0$, choose an integer $n$ such that $\alpha / n<b$; then

$$
\bar{S}_{-\alpha}=\bar{S}_{-\alpha / n}^{n}=\left(\bar{S}_{\alpha / n}^{-1}\right)^{n}=\left(e^{-(\alpha / n) A}\right)^{n}=e^{-\alpha A} .
$$

REMARK. If in the statement of Corollary 1 conditions (1), (2) and (4) but not (3) are satisfied, then by Theorem 1 there exist positive selfadjoint operators 
$A=\int_{0}^{\infty} \lambda d E(\lambda)$ and $B=\int_{0}^{\infty} \lambda d F(\lambda)$ such that $\bar{S}_{\alpha}=A^{\alpha}=\int_{0}^{\infty} \lambda^{\alpha} d E(\lambda)$ and $\bar{S}_{-\beta}=B^{\beta}$ $=\int_{0}^{\infty} \lambda^{\beta} d F(\lambda)$ for $0<\alpha<b$ and $0<\beta<-a$. If now $x \in D$, then

$$
\left(S_{\alpha} x, y\right)=\int_{0}^{\infty} \lambda^{\alpha} d(E(\lambda) x, y) \rightarrow(x, y)-(E(\{0\}) x, y)
$$

as $\alpha \rightarrow 0+$ and

$$
\left(S_{-\alpha} x, y\right)=\int_{0}^{\infty} \lambda^{\alpha} d(F(\lambda) x, y) \rightarrow(x, y)-(F(\{0\}) x, y)
$$

as $\alpha \rightarrow 0+$ for all $y \in D$. It follows, since $\alpha \rightarrow\left(S_{\alpha} x, y\right)$ is continuous on $a<\alpha<b$ for every $x$ and $y$ in $D$ (by (4)), that $E(\{0\}) x=F(\{0\}) x$ for all $x \in D$. Hence $E(\{0\})=F(\{0\})$. Let $P=E(\{0\}), \mathfrak{S}_{0}=R(P)$ and $\mathfrak{S}_{1}=\mathfrak{S}_{0}^{\perp}$. Then $\mathfrak{S}_{1}$ reduces each operator $\bar{S}_{\alpha}, a<\alpha<b$, and if $T_{\alpha}$ is the restriction of $\bar{S}_{\alpha}$ to $\mathfrak{S}_{1}$, then $\left\{T_{\alpha}\right\}, a<\alpha<b$, satisfies conditions (1)-(4) of Corollary 1. Hence there exists a unique selfadjoint operator $C$ in $\mathfrak{F}_{1}$ such that $T_{\alpha}=e^{\alpha C}$ for $a<\alpha<b$. The restriction of $\bar{S}_{\alpha}$ to $\mathfrak{S}_{0}$ is the zero operator $0_{0}$ in $\mathfrak{S}_{0}$. Thus, $\bar{S}_{\alpha}=0_{0} \oplus e^{\alpha C}$ for $a<\alpha<b$.

The next theorem differs from Theorem 1 only in that the interval of parameters is of the form $0<a<\alpha<\infty$, but this requires some basic changes at a few places in the proof. We omit the details.

THEOREM 2. Let $\left\{S_{\alpha}\right\}, 0<a<\alpha<\infty$, be a one-parametric family of symmetric operators in a Hilbert space $\mathfrak{S}_{\mathfrak{B}}$ and $D$ a dense linear manifold in $\mathfrak{S}$ such that

(1) $D \subset D\left(S_{\alpha} S_{\beta}\right)$ for all $a<\alpha, \beta$,

(2) $S_{\alpha} S_{\beta} x=S_{\alpha+\beta} x$ for all $x \in D$ and $a<\alpha, \beta$, and

(3) for every $x \in D,\left(S_{\alpha} x, x\right)$ is a bounded or Lebesgue measurable function of $\alpha$ in some interval.

Then the operators $S_{\alpha}, a<\alpha<\infty$, are essentially selfadjoint and there exists $a$ unique positive selfadjoint operator $T=\int_{0}^{\infty} \lambda d E(\lambda)$ such that

$$
\bar{S}_{\alpha}=T^{\alpha}=\int_{0}^{\infty} \lambda^{\alpha} d E(\lambda) \text { for } a<\alpha<\infty .
$$

4. Application. For an application of Theorem 1 consider a locally compact (Hausdorff) group $G$ with left-Haar measure $d x$ and a family of positive Radon measures $\left(\mu_{\alpha}\right), 0<\alpha<2 a$, on $G$ with the following properties (cf. [1]):

(1) $\mu_{\alpha}=\check{\mu}_{\alpha}$ for all $0<\alpha<2 a$ (i.e. the measures $\mu_{\alpha}$ are symmetric: $\int f(x) d \check{\mu}_{\alpha}(x)$ $\left.=\int f\left(x^{-1}\right) d \mu_{\alpha}(x)\right)$,

(2) $\mu_{\alpha} * \mu_{\beta}=\mu_{\alpha+\beta}$ for $0<\alpha+\beta<2 a$, and

(3) $\alpha \rightarrow \int f d \mu_{\alpha}$ is continuous on $0<\alpha<2 a$ for every $f \in C_{00}(G)$ (the space of continuous functions on $G$ with compact support).

Let $S_{\alpha}$, for $0<\alpha<a$, be the convolution operator $S_{\alpha} f=\mu_{\alpha} * f$ in $L^{2}=L^{2}(G, d x)$ with domain $\left\{f \in L^{2}\left|\mu_{\alpha} *\right| f \mid \in L^{2}\right\}$ and $D=C_{00}(G)$. Then one verifies easily that all conditions of Theorem 1 are satisfied and $T_{\alpha}=S_{\alpha}^{*}$ is the convolution operator $T_{\alpha} f=\mu_{\alpha} * f$ with maximal domain $\left\{f \in L^{2} \mid \mu_{\alpha} * f \in L^{2}\right\}$ for $0<\alpha<a$. Hence there exists by Theorem 1 a positive selfadjoint operator $A$ such that $T_{\alpha}=A^{\alpha}$ for $0<\alpha<a$. 
EXAMPLE. Let $G=R^{n}$ and $\mu_{\alpha}, 0<\alpha<n$, be the measures considered by $\mathrm{M}$. Riesz [4] in his theory of elliptic potentials of order $\alpha$ :

$$
\mu_{\alpha} * f(x)=\frac{1}{H_{n}(\alpha)} \int_{R^{n}} f(t)\|x-t\|^{\alpha-n} d t,
$$

where $H_{n}(\alpha)=\pi^{n / 2} 2^{\alpha} \Gamma(\alpha / 2) / \Gamma((n-\alpha) / 2)$. In this case $A=(-\Delta)^{-1 / 2}$, where $\Delta$ is the selfadjoint operator which is the closure of the Laplace operator in $L^{2}\left(R^{n}\right)$ with domain the $C^{\infty}$-functions with compact support. In this example condition (4) of Theorem 1 is satisfied so that $T_{\alpha}=e^{\alpha B}$, where $B=-\frac{1}{2} \log (-\Delta)$.

This well-known fact can be established using the theory of Fourier transform of distributions. For an arbitrary non-Abelian group $G$ no such tools are available in general.

\section{REFERENCES}

1. H. Cartan, Sur les fondements de la théorie du potentiel, Bull. Soc. Math. France 69 (1941), 71-96. MR 7, 447.

2. A. Devinatz, A note on semi-groups of unbounded self-adjoint operators, Proc. Amer. Math. Soc. 5 (1954), 101-102. MR 15, 632.

3. B. v. Sz. Nagy, Spektraldarstellung linearer Transformationen des Hilbertschen Raumes, Ergebnisse der Mathematik und ihrer Grenzgebiete, Band 5, Springer-Verlag, Berlin, 1942. MR 8, 276.

4. M. Riesz, L'intégrale de Riemann-Liouville et le problème de Cauchy, Acta Math. 81 (1949), 1-223. MR 10, 713.

5. D. V. Widder, The Laplace transform, Princeton Math. Series, vol. 6, Princeton Univ. Press, Princeton, N. J., 1941. MR 3, 232.

\section{WASHINGTON UNIVERSITY,}

ST. LouIs, Missouri 63130 